\title{
Component-resolved determination of the magnetization by magnetization-induced optical second-harmonic generation
}

\author{
Th. Gerrits and T. J. Silva \\ National Institute of Standards and Technology, Boulder, Colorado 80305 \\ Th. Rasing \\ Institute for Molecules and Materials, Radboud University Nijmegen, Toernooiveld 1, 6525 ED Nijmegen, The \\ Netherlands
}

(Received 29 September 2005; accepted 30 January 2006; published online 31 March 2006)

\begin{abstract}
We present a method for simultaneously measuring the two normalized in-plane magnetization components in magnetic thin films that allows for the reconstruction of the magnetization angle and the normalized value of the magnetization with subnanosecond temporal resolution. The calibrated method relies on the large contrast associated with magnetization-induced optical second-harmonic generation, permitting measurements with a dynamic range in excess of $25 \mathrm{~dB}$. Calibration is achieved by the determination of the relative magnitude and phase of the essential nonlinear optical tensor components, including those of a magneto-optic origin. Ellipsometric determination of the polarization of the second-harmonic signal, when the magnetization is saturated along four in-plane Cartesian directions, is sufficient to determine the second-order nonlinear tensor components. These four directions represent the two saturated states along the longitudinal and transverse directions of the magnetization, respectively. The tensor components are subsequently used to uniquely determine the in-plane magnetization amplitude and direction. (c) 2006 American Institute of Physics.
\end{abstract}

[DOI: $10.1063 / 1.2179414]$

\section{INTRODUCTION}

Separate determination of the components of the vector magnetization as a function of time in ferromagnetic films reveals basic features of the motion of the magnetization $\mathbf{M}$. For instance, the absolute value $|\mathbf{M}|$ of $\mathbf{M}$ can be obtained by vector summation of all the magnetization components. In time-domain studies, where the precessional free-induction decay of magnetization is measured, the generation of spin waves can result in a significant reduction of $|\mathbf{M}|{ }^{1}$ This particular information is important in precessional reversal studies of magnetic elements, as coherence during the magnetization motion is necessary if the precessional reversal is to be of practical utility. ${ }^{2}$ We suspect that various physical conditions promote incoherence of the switching event including the shape of the magnetic element and the uniformity of the applied magnetic fields. ${ }^{3,4}$

We have developed a method for a complete and unambiguous measurement of the normalized vector components of the in-plane magnetization in order to determine the evolution of $|\mathbf{M}|$ for time-resolved studies. However, only when $|\mathbf{M}|$ is conserved, the excitation angle of the magnetization can be determined unambiguously. Nevertheless, this method allows for the identification of spin waves by determining the normalized value of $|\mathbf{M}|$ from both normalized in-plane components of the magnetization. This technique can also be applied to study quasistatic reversal processes, such as hysteretic switching in poorly oriented materials, which are important for the study of exchange-bias effects. ${ }^{5}$ Of immediate interest is the use of such a technique for determining nonlinear mechanisms in the generation of spin waves during the switching process. ${ }^{1}$ A prerequisite for observing spin waves is the scale of the spatial resolution relative to the characteristic wavelength of the inhomogeneity in the magnetization. The wavelength of the excited spin waves (or domains in the static case) must be smaller than the optical resolution of the technique in question. In the present case, the resolution is approximately $5 \mu \mathrm{m}$. In addition, vector resolution is obtained only for the two in-plane components of $\mathbf{M}$. This is not a significant limitation, because the precessional motion of magnetization in thin magnetic films with in-plane anisotropy and weak uniaxial anisotropy manifests only a small out-of-plane component of the order of $1^{\circ}-2^{\circ}$.

Magnetization-induced optical second-harmonic generation (MSHG) is used to measure the two in-plane components of the magnetization. ${ }^{6}$ MSHG has already been used successfully for extraction of the magnetization vector by simultaneously measuring the second-harmonic polarization rotation and the second-harmonic intensity. ${ }^{6}$ These studies presumed a quasistatic coherent magnetization rotation along the hard axis when determining the calibration values for the absolute value of $|\mathbf{M}|$. MSHG offers large contrasts, of the order of $50 \%$, for Permalloy $\left(\mathrm{Ni}_{80} \mathrm{Fe}_{20}\right)$ which facilitates artifact-free calibration of the magnetization components. However, the efficiency of the second-harmonic signal is rather small, requiring large integration times to obtain a sufficient signal to noise ratio.

We present here a calibration method that allows us to determine the normalized magnetization vector components for a thin Permalloy film using saturated magnetization states. Permalloy is a suitable candidate for these studies, as its reversal mechanism has been extensively studied for ap- 
plications in tunneling magnetoresistance (TMR) and giant magnetoresistance (GMR) memory devices. Furthermore, Permalloy provides large MSHG contrasts. ${ }^{7}$ Several other groups have tried to measure vector- and time-resolved magnetization dynamics. Acreman et $a l^{8}$ used the linear magneto-optical Kerr effect (MOKE) to probe a componentresolved trajectory of $\mathbf{M}$. In this case the excitation was small so that one component of $\mathbf{M}$ could be assumed to be of constant magnitude. A calibration of the two other components was not achieved. One fundamental problem of the magneto-optical Kerr effect is that the cross talk between the two magnetization components is always present in timeresolved studies, because polarization rotation originates from both the polar component and the longitudinal component of the magnetization. In the linear case, the rotation due to the relatively high sensitivity of the polar effect is not negligible, even when magnetization rotations of the in-plane components of $\mathbf{M}$ are very large and the out-of-plane motion is very small. To separate both the longitudinal and polar magnetization components, Choi et al. ${ }^{9}$ employed a quadrant detection scheme. This method allows measuring all three components of the magnetization. However, due to the small dynamic range in the linear contrast, calibration of the vector components of $\mathbf{M}$ is difficult. Nevertheless, linear magnetooptic studies do not need photon-counting systems and are therefore much easier to work with. A separation of the longitudinal and polar components was also reached by Ding et $a l .{ }^{10}$ By changing the incident light path, the experimental geometry was changed and the separation was reached by two independent measurements. Lee et al. ${ }^{11}$ demonstrated vector resolution with linear MOKE using $s$ - and $p$-incident waves and by changing the orientation of the applied magnetic field. A similar approach was presented by Lopusnik. ${ }^{12}$ It was shown that by subtracting and adding two independent magneto-optical signals with opposite but equal bias fields or pulse fields separate the polar and longitudinal magnetization components. The later three methods are based on several independent measurements in order to reconstruct the individual components of the magnetization. Kabos et al. ${ }^{6}$ used MSHG to simultaneously probe the two in-plane magnetization components. Calibration in the latter experiment was performed on the basis of the Stoner-Wohlfarth behavior of magnetic thin films and subsequently fitting the magnetization dependencies to the model. Our approach is similar to that applied by Kabos et al. but differs in that the calibration is based on well defined saturated states of the magnetization, which are independent of the details of the magnetization reversal process and which make the calibration more general and reliable for different materials.

\section{MSHG FROM A MAGNETIZED SURFACE}

In the dipole approximation, the polarization of the light field interacting with a medium under high intensity optical illumination can be expressed by a Taylor series: ${ }^{13}$

$$
\mathbf{P}=\chi^{(1)} \mathbf{E}+\chi^{(2)} \mathbf{E} \mathbf{E}+\chi^{(3)} \mathbf{E} \mathbf{E} \mathbf{E} \ldots,
$$

where $\chi^{(n)}$ is the susceptibility tensor of $n$th order and rank $n+1$. The generation of optically induced second-harmonic light is described by the second term in Eq. (1). The second-

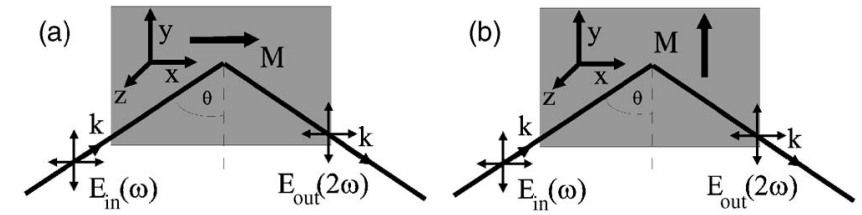

FIG. 1. Geometries for MSHG. (a) Longitudinal configuration in which $\mathbf{M}$ lies parallel to the plane of incidence and (b) transverse configuration, with $\mathbf{M}$ perpendicular to the plane of incidence.

harmonic polarization of the medium is given by

$$
P_{i}(2 \omega)=\chi_{i j k}^{(2)} E_{j}(\omega) E_{k}(\omega) .
$$

The third rank tensor $\chi_{i j k}^{(2)}$ in Eq. (2) consists of 27 elements. However, as the electric field indices $j$ and $k$ can be interchanged for a single beam experiment, the number of independent elements is reduced to 18 .

Figure 1 shows the configuration for the two in-plane components of $\mathbf{M}\left(M_{x}, M_{y}\right)$ in the MSHG experiment. For the longitudinal configuration $\left(M_{x}\right)$, the tensor for an isotropic surface is given by ${ }^{14}$

$\chi^{(2)}\left(M_{x}\right)$

$$
=\left(\begin{array}{cccccc}
0 & 0 & 0 & 0 & \chi_{x z x}^{(\text {even })} & \chi_{x y x}^{\text {(odd) }} \\
\chi_{y x x}^{\text {(odd) }} & \chi_{y y y}^{\text {(odd) }} & \chi_{y z z}^{\text {(odd) }} & \chi_{y y z}^{(\text {even })} & 0 & 0 \\
\chi_{z x x}^{\text {(even) }} & \chi_{z y y}^{\text {(even) }} & \chi_{z z z}^{\text {(even) }} & \chi_{z y z}^{\text {(odd) }} & 0 & 0
\end{array}\right),
$$

where $\chi_{i j k}^{\text {(odd) }}$ represents a tensor element that undergoes a phase change of $\pi$ (i.e., changes sign) upon reversal of $M_{x}$, whereas all $\chi_{i j k}^{\text {(even) }}$ are invariant upon reversal of $M_{x}$.

For the transverse configuration $\left(M_{y}\right)$, the tensor is $\chi^{(2)}\left(M_{y}\right)$

$$
=\left(\begin{array}{cccccc}
\chi_{x x x}^{\text {(odd) }} & \chi_{x y y}^{\text {(odd) }} & \chi_{z z z}^{\text {(odd) }} & 0 & \chi_{x z x}^{\text {(even) }} & 0 \\
0 & 0 & 0 & \chi_{y y z}^{(\text {(even })} & 0 & \chi_{y x y}^{(\text {odd })} \\
\chi_{z x x}^{(\text {(even) }} & \chi_{z y y}^{(\text {even })} & \chi_{z z z}^{(\text {even) }} & 0 & \chi_{z z x}^{\text {(odd) }} & 0
\end{array}\right) .
$$

For the case of precessional magnetization dynamics in magnetic thin films with weak applied fields, the time-varying polar magnetization component remains small and its contribution to the MSHG signal can safely be ignored. A more effective means to probe the out-of-plane component of $\mathbf{M}$ can be achieved through the use of the polar magneto-optical Kerr effect. ${ }^{2}$

The above tensors constitute the complete set of MSHG tensor components for isotropic surfaces and can be used to analyze the second-harmonic polarization due to a magnetization state. The overall response can be further simplified by summing over all even and odd tensor components for a given incident electromagnetic field. If the incident field is $p$ polarized $\left(E_{p}^{2}=E_{x}^{2}+E_{z}^{2}\right)$, the polarization generated by the magnetized surface $\left(E_{\text {out }}\right)$ is given by

$$
\mathbf{P}(2 \omega)=\left(\begin{array}{c}
\chi_{x x x}^{\text {(odd) }} E_{x} E_{x}+\chi_{x z z}^{\text {(odd) }} E_{z} E_{z}+\chi_{x z x}^{(\text {even })} E_{z} E_{x} \\
\chi_{y x x}^{\text {(odd) }} E_{x} E_{x}+\chi_{y z z}^{\text {(odd })} E_{z} E_{z} \\
\chi_{z x x}^{\text {(even) }} E_{x} E_{x}+\chi_{z z z}^{\text {(even })} E_{z} E_{z}+\chi_{z z x}^{\text {(odd })} E_{z} E_{x}
\end{array}\right) .
$$

A summation over all odd and even tensor elements and representation of $\mathbf{E}$ in terms of the Jones matrix calculus yields 


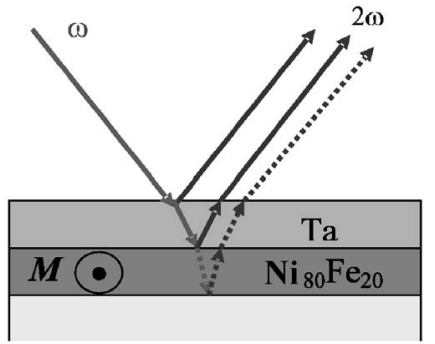

FIG. 2. Magnetization-induced second-harmonic generation at each interface: air-tantalum, tantalum-Permalloy, and Permalloy-nonmagnet.

$$
\begin{aligned}
\mathbf{E}\left(2 \omega, m_{x}, m_{y}\right) & =\left(\begin{array}{c}
E_{s}(2 \omega) \\
E_{p}(2 \omega)
\end{array}\right) \\
& \propto\left(\begin{array}{c}
\chi_{l}^{\text {(odd })} e^{i \phi_{l} m_{x}} \\
\chi^{\text {(even) }}+\chi_{t}^{\text {(odd })} e^{i \varphi_{t} m_{y}}
\end{array}\right) E_{p}^{2}(\omega),
\end{aligned}
$$

where $m_{x}$ and $m_{y}$ are the normalized in-plane magnetization values; $E_{s}=E_{y}, E_{z}=E_{p} \sin \theta, E_{x}=E_{p} \cos \theta$, where $\theta$ is the angle of incidence; and $\chi_{l / t}^{\text {(odd) }}$ and $\chi^{\text {(even) }}$ are the sum of the odd (both longitudinal and transverse) and even tensor components, respectively. In Eq. (6) $\phi_{l}$ and $\phi_{t}$ are the relative phases between the even and odd generalized tensor elements for the longitudinal and transversal cases, respectively. Note that these tensor elements are generalized tensor elements, because they also include the individual Fresnel coefficients and therefore strongly depend on the angle of incidence. The dependence on the magnetization state is taken into account by considering the relative magnetization components. In the following derivation, a birefringent effect from the individual sample layers is not taken into account. We did not observe any induced birefringence during polarization measurements. However, the use of sapphire substrates may unintentionally lead to birefringence, thus rotating the emitted second-harmonic light. In that case, birefringence has to be taken into account. The emitted second-harmonic intensity is obtained from the square of the magnitude of the complex second-harmonic field: $I=E E^{*}$.

The simple representation in Eq. (6) describes the magnetization dependence of the second-harmonic response. A nonmagnetic interface contribution is present only in the $p$ component. However, the magnetic contribution represented as $\chi^{\text {(odd) }} e^{i \phi}$ depends directly on the magnetization and affects both the $s$ and $p$ components of the induced polarization.

Figure 2 shows an example of a multilayer structure. The structure consists of a nonmagnetic layer, which is optically thick. ${ }^{15}$ On the top of this layer, a thin $(50 \mathrm{~nm})$ Permalloy layer is deposited and capped with a thin $(5 \mathrm{~nm})$ Ta layer to prevent surface oxidation of the Permalloy. The incident light field traverses through the various layers. A secondharmonic signal is generated at each interface. The overall MSHG signal is given by the coherent superposition of signals coming from all individual interfaces. As a result, interference leads to either enhancement or cancellation of the magnetic contrast, depending on the individual interfaces and layer thicknesses. ${ }^{16}$ If the magnetic layer is chosen to be

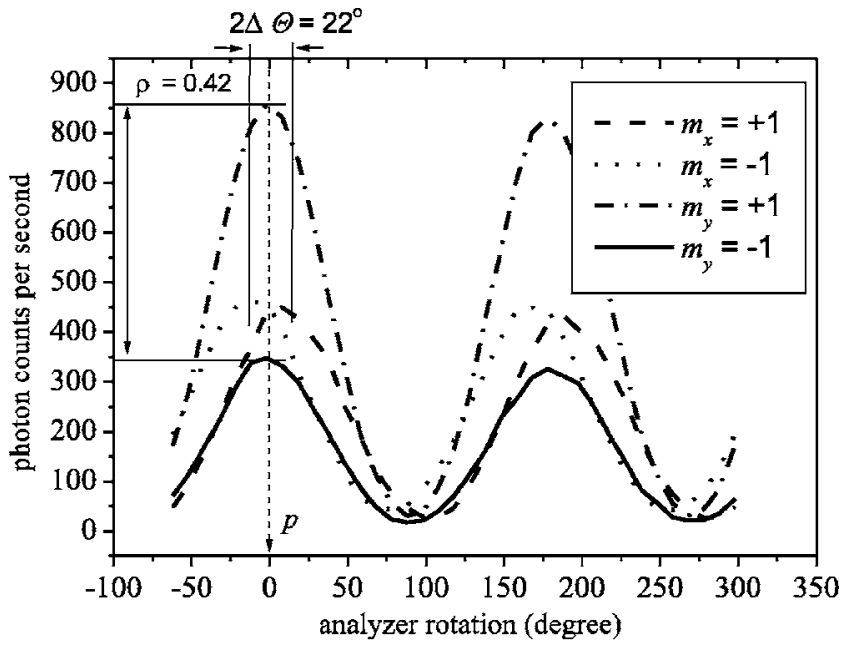

FIG. 3. Polarization of the second-harmonic generated light as a function of the magnetization state. In the case of a longitudinal magnetization, the second-harmonic polarization rotates (dotted and dashed curves) with respect to the incident polarization $(p)$, as predicted by Eq. (6). Transverse magnetization changes the intensity (the dash-dotted and solid curves).

optically thick such that no fundamental light reaches the bottom interface, the magnetic signal only originates from the top magnetic interface.

Figure 3 shows data for the magnetization-dependent measurement of the second-harmonic polarization for this multilayer structure, saturated either perpendicular or parallel to the plane of incidence. The polarization state is measured by rotating an analyzer in the path of the generated secondharmonic light. In this particular case of $p$-polarized incident light, a longitudinally oriented magnetization $\left(m_{x}\right)$ rotates the outgoing second-harmonic polarization with respect to the incident field. The transverse magnetization $\left(m_{y}\right)$ changes the overall second-harmonic yield, resulting in changes of the second-harmonic intensity. Hence, separating the intensity change and polarization rotation allows us to separate the two in-plane components of $\mathbf{M}$. The contrast in the MSHG signal is $42 \%$ for the transverse component of the magnetization and $\pm 11^{\circ}$ for the longitudinal component.

\section{CALIBRATION OF THE MAGNETIZATION COMPONENTS}

The experimental setup for measuring and calibrating an in-plane, vector-resolved magnetization motion is given in Fig. 4. A high intense $\left(\sim 100 \mathrm{GW} / \mathrm{cm}^{2}\right)$ femtosecond laser pulse is focused down to a spot size of about $5 \mu \mathrm{m}$ in diameter by means of a microscope objective (MO) lens. A polarizer $(P)$, in front of the microscope objective, is used to choose the incoming polarization parallel to the plane of incidence ( $p$ polarization). A second microscope objective serves as a collimator for the generated second harmonic. Due to the high laser intensities, the second-harmonic light is potentially generated at each optical component. By placing an infrared filter in front of the sample, we measured the second-harmonic intensity generated by all optical components in the setup. These measurements did not show any significant second-harmonic signal (close to the dark count). Therefore, the second-harmonic intensity measured is only 


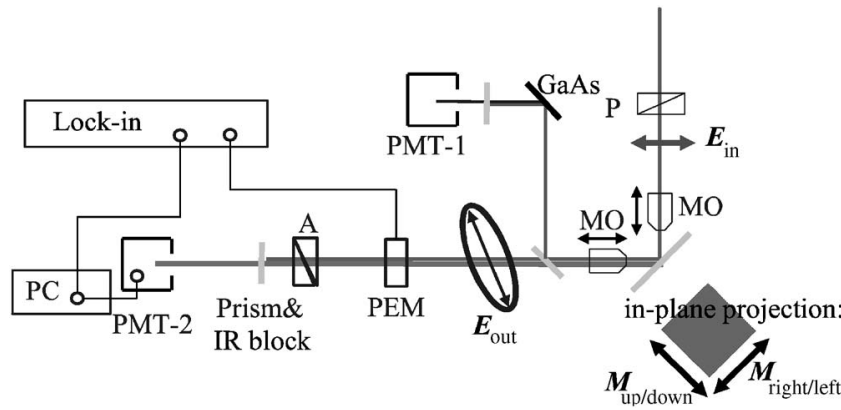

FIG. 4. The experimental configuration used to probe the two in-plane components of $\mathbf{M}$. The average intensity is measured at the photomultiplier tube (PMT). The polarization rotation is determined by modulation with a photoelastic modulator and measurement of the modulation signal by means of a lock-in amplifier. Note that in this figure the sample is rotated by $90^{\circ}$ to show the various magnetization directions.

generated at the sample. The polarization state of the secondharmonic light is determined by the magnetization state of the sample and is represented by the rotated ellipse. Our goal is to simultaneously measure both in-plane magnetization components. ${ }^{6}$ To achieve this, the second-harmonic signal is split into two parts with different types of signal processing. A photoelastic modulator (PEM) is employed to (a) compensate intensity changes due to polarization rotations originating from the sample and (b) quantitatively measure the polarization rotation. The result is that the photomultiplier signal, being one branch of the signal processing, is sensitive only to $m_{y}$, whereas a separate signal path uses a lock-in amplifier to detect $m_{x}$ by measuring the amplitude of modulation induced by the PEM. The PEM retardation axis is aligned along the direction of $p$ polarization. Determination of the generalized tensor components in Eq. (6) is done by measuring the second-harmonic signal when the magnetization is saturated along four different directions. These directions are given by $m_{\mathrm{up}}$ and $m_{\mathrm{down}}$ in the case of $m_{y}$, and $m_{\text {right }}$ and $m_{\text {left }}$ for $m_{x}$. The values determined for the generalized tensor components are subsequently used for the calibration of the magnetization component measurements.

For the case where the analyzer is operating in the $p$ direction and with $\mathbf{m}$ switching between the two saturated states $m_{y}= \pm 1$, the corresponding second-harmonic efficiencies are

$$
\begin{aligned}
\frac{I(2 \omega)}{I^{2}(\omega)}= & \left|\chi^{(\text {even })}+\chi_{t}^{(\text {odd })} m_{y} e^{i \varphi_{t}}\right|^{2}=\left|\chi^{(\text {even })}\right|^{2}+\left|\chi_{t}^{(\text {odd })}\right|^{2} m_{y}^{2} \\
& +\left|\chi^{(\text {even })}\right|\left|\chi^{(\text {even })}\right| m_{y} \cos \varphi_{t}
\end{aligned}
$$

Equation (7) contains three unknowns: $\chi^{(\text {even })}, \chi_{t}^{\text {(odd) }}$, and $\phi_{t}$. In Eq. (7), $I^{2}(\omega)$ is determined by an additional optical detection branch that measures the second-harmonic intensity due to reflection from a GaAs reference. The intensity difference for the two magnetization states of $m_{y}$ (up and down) is proportional to $4 \chi_{t}^{\text {(odd) }} \chi^{\text {(even) }} \cos \phi_{t}$. Its sum is proportional to $2\left(\left[\chi^{(\text {even })}\right]^{2}+\left[\chi_{t}^{\text {(odd })}\right]^{2}\right)$. The term $\left[\chi^{(\text {even })}\right]^{2}$ can be obtained by saturating $\mathbf{m}$ along the $x$ axis and setting the analyzer to $p$ [see Eq. (6)]. Using this method, we obtain $\chi^{\text {(even) }}, \chi_{t}^{\text {(odd) }}$, and $\phi_{t}$.

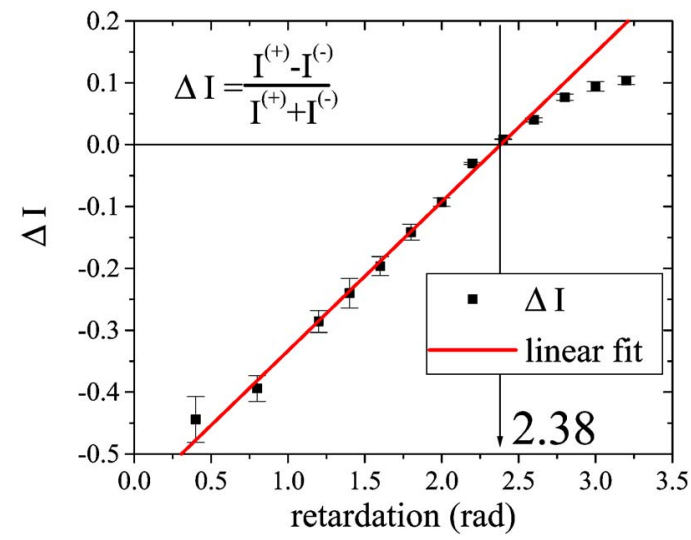

FIG. 5. (Color online) The change in intensity is plotted as a function of the photoelastic modulator retardation. The magnetization is switched between the two saturated states of $M_{x}\left(M_{\text {left }}\right.$ and $\left.M_{\text {right }}\right)$.

Additional measurements are required to determine the remainder of the generalized tensor components $\chi_{l}^{\text {(odd) }}$ and $\phi_{l}$. These values are obtained by similar addition and subtraction of the two signals for an analyzer setting of $45^{\circ}$. However, as will be shown later, these parameters are not actually necessary in order to calibrate the magnetization components.

To determine $m_{y}$, we require values for the parameters $\chi^{(\mathrm{even})}, \chi_{t}^{\text {(odd) }}$, and $\phi_{t}$. From Eq. (6) we see that the average MSHG intensity $\left(I=E E^{*}\right)$ not only depends on the transverse component of $\mathbf{m}$ but also on the longitudinal magnetization component. The latter rotates the outgoing electromagnetic field, resulting in a change of the electric field projection along the analyzer axis. The average intensity, however, should only be affected by $M_{y}$. Hence, the cross talk between the signals corresponding to the two magnetization components exists when the average intensity changes upon polarization rotation. However, this intensity change can be compensated using a PEM. In the present configuration, where the PEM crystal axis is set along $p$ and the analyzer is set to $45^{\circ}$, a PEM retardation of 2.407 rad is sufficient to compensate for the induced intensity changes due to a small rotation of the MSHG polarization. Figure 5 provides a proof of concept for this approach, and the data clearly show the intensity difference as a function of PEM retardation for the case where the magnetization is switched in the longitudinal direction. This measurement was performed to obtain the real zero crossing, which in this case is $2.38 \mathrm{rad}$.

Using Jones matrices, the electric field at the PMT is given by

$$
\begin{aligned}
& \mathbf{E}\left(2 \omega, m_{x}, m_{y}\right) \propto \frac{1}{\sqrt{2}}\left(\begin{array}{ll}
1 & 1 \\
1 & 1
\end{array}\right)\left(\begin{array}{c}
\chi_{l}^{\text {(odd) }} e^{i \phi_{l} m_{x}} \\
\chi^{(\text {even })}+\chi_{t}^{\text {(odd) }} e^{i \varphi_{t}} m_{y}
\end{array}\right)
\end{aligned}
$$

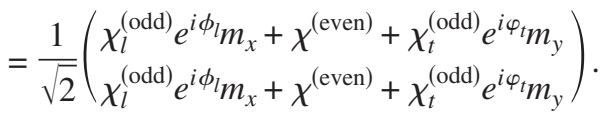

As the PEM retardation is chosen to compensate any intensity variations, due to changes in $m_{x}$, the second-harmonic intensity $\left(I=E E^{*}\right)$ is given by 


$$
\begin{aligned}
I(2 \omega)= & \frac{1}{2}\left[\left|\chi^{(\text {even })}\right|^{2}+\left|\chi_{t}^{(\text {odd })}\right|^{2} m_{y}^{2}+2\left|\chi^{(\text {even })}\right|\right. \\
& \left.\times\left|\chi^{(\text {even })}\right| m_{y} \cos \varphi_{t}\right] I^{2}(\omega) .
\end{aligned}
$$

Solving Eq. (9) for $m_{y}$ using the measured values for $\chi^{(\mathrm{even})}$, $\chi_{t}^{\text {(odd) }}$, and $\phi_{t}$ yields the transverse magnetization component

$$
m_{y}=-\frac{\left|\chi^{\text {(even) }}\right|}{\left|\chi_{t}^{\text {(odd) }}\right|} \cos \varphi_{t} \pm \frac{\sqrt{2 I(2 \omega)}}{I(\omega)\left|\chi_{t}^{\text {(odd) }}\right|} .
$$

Measurement of $m_{x}$ requires calibration of the lock-in signal at the two saturated states for the longitudinal component $\left(m_{x}\right)$. The lock-in signal $(S)$ is equal to a conversion coefficient $(K)$ times the average second-harmonic intensity $[I(2 \omega)]$ and polarization-rotation angle $\theta$ :

$$
S=K I(2 \omega) \theta,
$$

where $\theta$ can be represented by a real and imaginary part $\theta_{r}$ $+i \theta_{e}$, where $\theta_{r}$ is the polarization rotation and $\theta_{e}$ is the ellipticity. The polarization-rotation angle is given by

$$
\begin{aligned}
\theta & =\arctan \left[\frac{\chi_{l}^{\text {(odd) }} e^{i \phi_{l} m_{x}}}{\chi^{\text {(even) }}+\chi_{t}^{\text {(odd) }} e^{i \phi_{t} m_{y}}}\right] \\
& \approx \frac{\chi_{l}^{\text {(odd) })} e^{i \phi_{l} m_{x}}}{\chi^{(\text {even })}+\chi_{t}^{\text {(odd) }} e^{i \phi_{t} m_{y}}},
\end{aligned}
$$

where we have used the approximation of $\arctan (\theta) \sim \theta$ for small rotation angles. Combining Eqs. (11) and (12), we obtain

$$
m_{x}=\frac{S}{K I(2 \omega)}\left(\frac{\chi^{(\text {even })}+\chi_{t}^{\text {(odd) }} e^{i \phi_{t} m_{y}}}{\chi_{l}^{\text {(odd) }} e^{i \phi_{l}}}\right) .
$$

In the case of a longitudinally saturated sample $\left(m_{x}= \pm 1\right.$, $m_{y}=0$ ), Eqs. (11) and (12) yield

$$
\theta_{0}=\frac{S_{0}}{K I_{0}(2 \omega)} \approx \pm \frac{\chi_{l}^{\text {(odd) }} e^{i \phi_{l}}}{\chi^{\text {(even) }}},
$$

where $K$ is determined by measuring the corresponding intensity $I_{0}(2 \omega)$ and the lock-in signal $S_{0}$. By combining Eqs. (13) and (14), $m_{x}$ can be determined:

$$
m_{x}=\frac{S I_{0}(2 \omega)}{S_{0} I(2 \omega)}\left(\frac{\chi^{(\mathrm{even})}+\chi_{t}^{(\mathrm{odd})} e^{i \phi_{t} m_{y}}}{\chi^{(\mathrm{even})}}\right) .
$$

Note that when extracting $m_{x}$ from the data, the magnetic tensor component $\chi_{l}^{\text {(odd) }}$ and phase $\phi_{l}$ do vanish, owing to the calibration procedure used.

\section{THE INFLUENCE OF JITTER}

Jitter must be accounted for in time-resolved electrical pump-optical probe experiments. In the case of optically generated magnetic field pulses, ${ }^{2}$ the jitter is on the order of fluctuations in the beam path (a few femtoseconds). However, an electronically generated magnetic field pulse has a non-negligible uncertainty of arrival with respect to the arrival of the probe laser pulse. In our time-resolved experiment, this jitter was $50 \mathrm{ps}$. Such uncertainty gives rise to errors in the determination of $|\mathbf{M}|$. The measured value for $|\mathbf{M}|$, including uncertainty $\Delta \Psi$ in the excitation angle $\Psi$, is given by

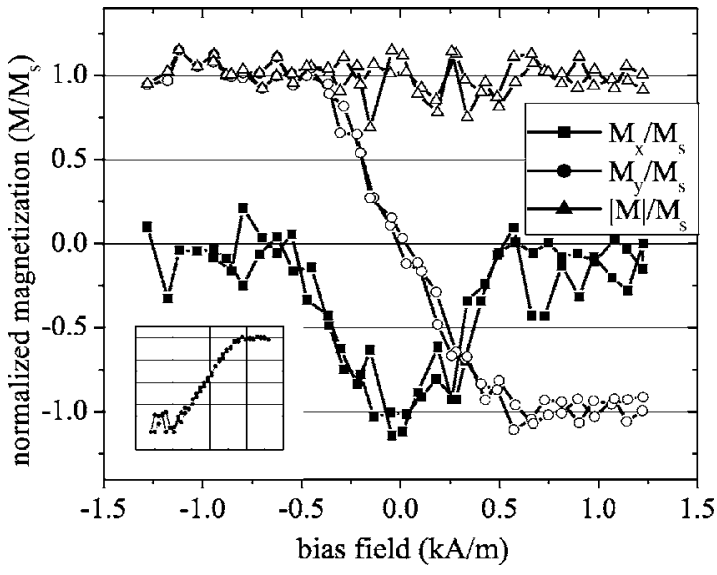

FIG. 6. Two hard-axis dependencies as measured by MSHG, at which a prior calibration was performed to calculate the in-plane magnetization components from the data for a sample with low contrast and large contrast (inset). The low contrast measurements were performed on a $\mathrm{Ta}_{5} \mathrm{NiFe}_{50} \mathrm{Cu}_{5}$ multilayer on glass, where the numbers indicate the individual layer thickness in nanometer. The high contrast measurements were performed on a $\mathrm{Ta}_{1} \mathrm{NiFe}_{50} \mathrm{Cu}_{5}$ multilayer on sapphire. Both measurements were done by probing through the substrates.

$$
\begin{aligned}
|\mathbf{M}|^{2} & =\left(M_{x}^{2}+M_{y}^{2}\right)=M_{S}^{2}\left[\cos ^{2}(\Psi+\Delta \Psi)+\sin ^{2}(\Psi+\Delta \Psi)\right] \\
& =M_{S}^{2} \cos ^{2} \Delta \Psi .
\end{aligned}
$$

Hence, the determination of $|\mathbf{M}|$ depends on the temporal $(\Delta \tau)$ uncertainty of the excitation angle. As a first order approximation of this error, we write $\Delta \Psi$ as

$$
\Delta \Psi=\frac{\partial \Psi}{\partial t} \Delta \tau .
$$

Assuming a Gaussian distribution in the jitter $\rho\left(t^{\prime}\right)$, the reconstructed absolute value of $\mathbf{M}$ at time $t=t_{0}$ becomes

$$
\begin{aligned}
\left|\mathbf{M}\left(t_{0}\right)\right|^{2} & =2 \int_{0}^{\infty}\left|\mathbf{M}\left(t^{\prime}\right)\right| \rho\left(t^{\prime}\right) d t^{\prime} \\
& =2 M_{S}^{2} \int_{0}^{\infty} \rho\left(t^{\prime}\right) \cos ^{2}\left(\left.\frac{\partial \Psi}{\partial t}\right|_{t=t_{0}} t^{\prime}\right) d t^{\prime},
\end{aligned}
$$

where $\rho(t)=(1 / \sqrt{2 \pi \sigma}) e^{-t^{2} / 2 \sigma^{2}}$. Equation (18) shows that the reduction of $|\mathbf{M}|$ increases with increasing $\partial \Psi / \partial t$. For largeangle motions, this implies that such an apparent reduction of $|\mathbf{M}|$ is stronger. In the case of our experiments, we calculate that the maximum reduction of $|\mathbf{M}|$, due to temporal uncertainty in the arrival of the excitation pulse, was only $3 \%$ due to 50 ps of jitter. Such a small reduction of $|\mathbf{M}|$ is within the noise of our present experiments.

\section{APPLICATION OF VECTOR-RESOLVED MEASUREMENTS}

The validity of the above measurement scheme will be demonstrated by a simple coherent quasistationary rotation of the magnetization and by a time-resolved measurement of the magnetization after excitation with a short magnetic field pulse.

Figure 6 shows the two in-plane components as well as the magnitude of the normalized magnetization for an external field along the in-plane hard axis of a thin Permalloy 


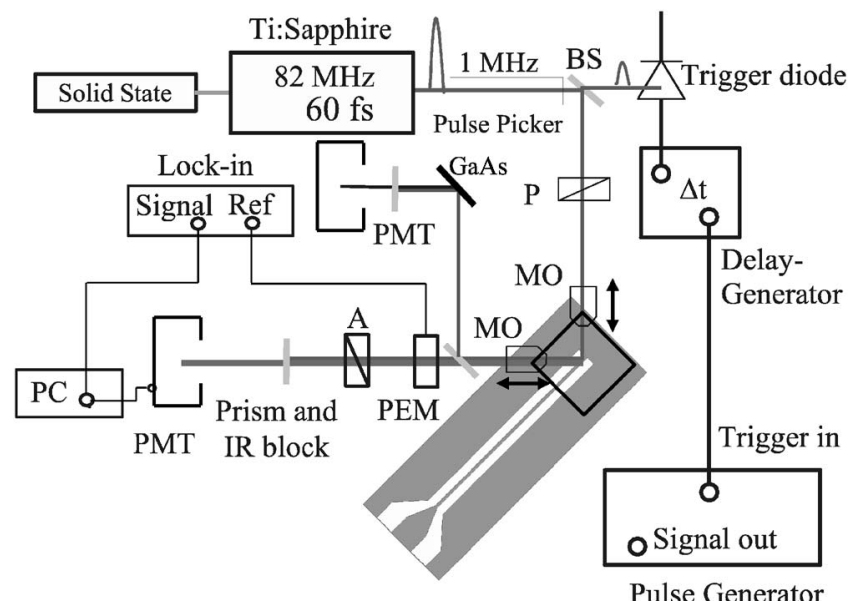

FIG. 7. Experimental pump-probe setup. The magnetic pulse field is generated by an electronic pulse generator. The time delay between pump and probe is controlled by a delay generator.

film. After performing the calibration, the two normalized in-plane components of $\mathbf{M}$ could be measured during coherent in-plane rotation. The figure shows the $x$ component, the $y$ component, and the calculated normalized absolute value of $\mathbf{M}$. The absolute value of $\mathbf{M}$ stays constant upon reversal of the magnetization and the obtained hard-axis response shows a dominant Stoner-Wohlfarht behavior of the magnetization. Though the demonstration is very convincing, a drawback of the method should still be mentioned. The present sample was a $50 \mathrm{~nm}$ Permalloy thin film, covered by $5 \mathrm{~nm}$ tantalum that significantly reduced the magnetic contrast and therefore the signal to noise ratio for the magnetic signal. The inset of the figure shows a hard-axis dependence that was obtained from a magnetic thin film with a $1 \mathrm{~nm}$ cover layer of tantalum. Note that the contrast reversed sign as compared to the thick capping layer. The magnetic contrast is also significantly increased and so is the signal to noise ratio. However, at small signal amplitudes, the noise starts to dominate, which is seen in the hard-axis loop for negative fields. The signal to noise ratio for positive bias fields is much better. Therefore, the experimental scheme can preferentially be used to probe magnetization dynamics for small excitation angles in a region where the signal to noise ratio is large. On the other hand, a small magnetic contrast makes the term $\left|\chi_{t}^{\text {(odd) }}\right|^{2} m_{y}^{2}$ in Eq. (9) negligible and the signal depends linearly on $\mathbf{M}$. However, in this case the integration time has to be much longer in order to obtain the same signal to noise ratio.

We will now discuss the application of the above technique to determine the magnetization dynamics, after excitation with a steplike magnetic field pulse, using the setup shown in Fig. 7. The measurement was made using a $50 \mathrm{~nm}$ Permalloy thin film grown on a glass coverslip and covered with a $1 \mathrm{~nm}$ Ta capping layer. The temporal resolution of the experimental data is $10 \mathrm{ps}$, being the minimum adjustable time delay of the delay generator, whereas the temporal accuracy was only $50 \mathrm{ps}$, given the jitter in the pulse generation electronics discussed earlier. Figure 7 shows the experimental configuration that was used to perform the vectorresolved pump-probe experiment. A voltage pulse generator
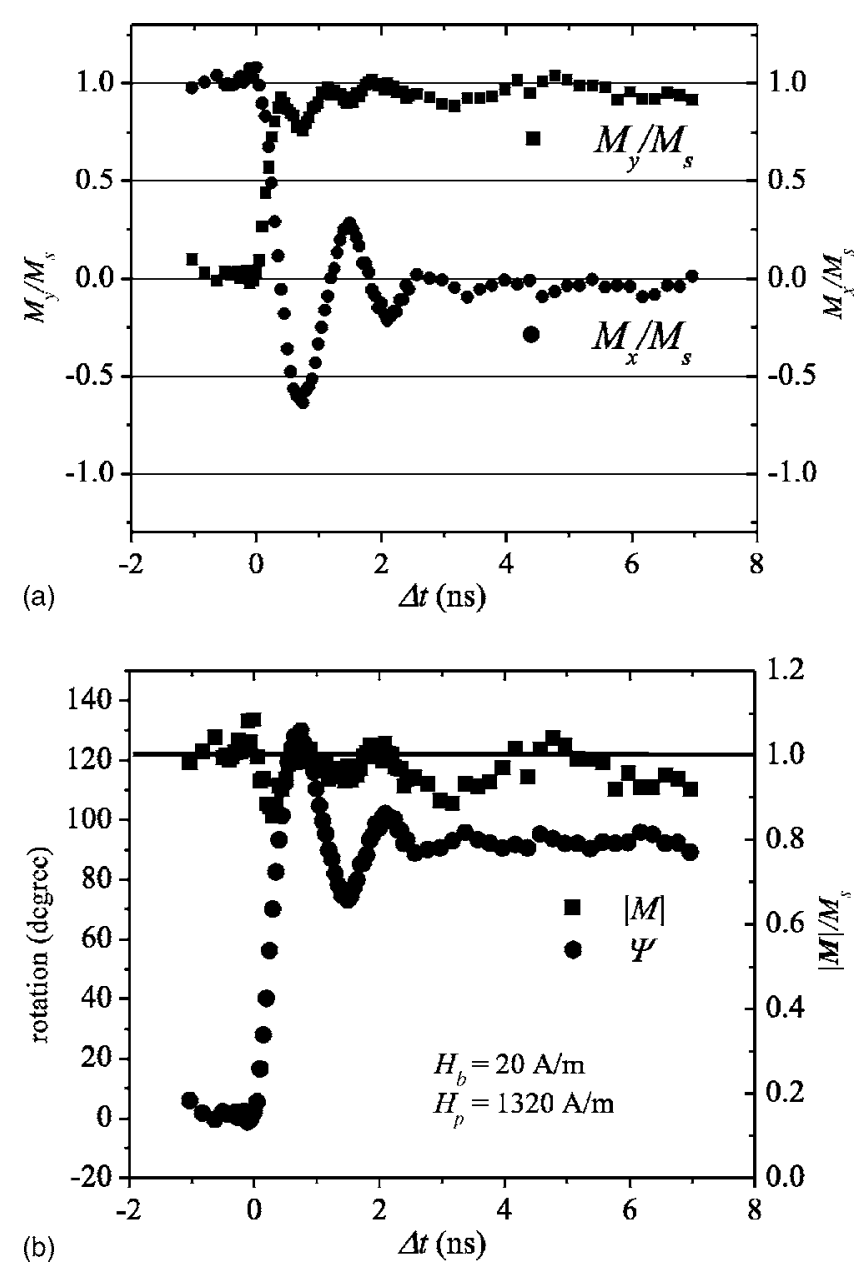

FIG. 8. (a) Calibrated in-plane vector components of the magnetization motion after excitation with a steplike $1.23 \mathrm{kA} / \mathrm{m}$ magnetic field pulse. The bias field was $20 \mathrm{~A} / \mathrm{m}$. (b) Reconstruction of the excitation angle (circles) and absolute value (squares) of the magnetization from the two in-plane components shown in (a).

was used to excite the magnetic thin film by the use of a coplanar waveguide (CPW) upon which the sample was placed. The magnetic fields produced at the CPW surface were steplike field pulses with an amplitude of $1.23 \pm 0.02 \mathrm{kA} / \mathrm{m}(15.5 \pm 0.2 \mathrm{Oe})$, a rise time of about $50 \mathrm{ps,}$ and a duration of $10 \mathrm{~ns}$. The actual data are taken only at the leading edge of the magnetic field pulse representing a delay of about $7 \mathrm{~ns}$. The CPW used for the measurements has a center conductor width of $130 \mu \mathrm{m}$ and a $50 \Omega$ characteristic impedance, which minimizes loss. The CPW surface is coated with a 1-2 $\mu \mathrm{m}$ insulating layer of polyimide. The magnetic sample is placed on the top of the CPW structure with the magnetic film laid down such that the sample is in the closest possible proximity to the CPW surface. The applied bias field $H_{b}=20 \mathrm{~A} / \mathrm{m}(0.2 \mathrm{Oe})$ is aligned parallel to the anisotropy easy axis of the magnetic thin film along the $x$ axis, thus also being the initial direction of $\mathbf{m}$. The laser spot was focused to a diameter of approximately $5 \mu \mathrm{m}$. The incident polarization of the laser pulse was set to $p$. Both the amplitude and polarization rotation of the MSHG signal were analyzed using the previously described method. Figure 8(a) shows data for the two experimentally determined inplane magnetization components $m_{x}$ and $m_{y}$. The calculated 
magnetization angle and the absolute value of $\mathbf{m}$ are presented in Fig. 8(b). The dip in the plots of $|\mathbf{m}|$ can be partly explained in terms of jitter. As for the extracted excitation angle, we observed a maximum rotation of $135^{\circ}$. At that point in time and at maximum magnetization change, there is no significant reduction in $|\mathbf{m}|$ after the subtraction of the jitter contribution. This leads us to conclude that the spatial coherence of the magnetization motion is preserved even at high excitation angles, to within the uncertainty of our measurement technique. ${ }^{2}$ These results confirm previous observations of the uniformity of large-angle magnetization dynamics when the initial direction of the magnetization is aligned along the easy-axis direction of the uniaxial induced anisotropy. ${ }^{1}$

\section{DISCUSSION}

We have demonstrated a vector-resolved measurement of the magnetization vector based on the nonlinear optical technique of magnetization-induced second-harmonic generation (MSHG). A general calibration method was presented that may be used for arbitrary materials to measure the in-plane components of $\mathbf{M}$, albeit by neglecting a polar contribution in the polarization rotation in the case of weak applied magnetic fields. This method does not presume any detailed physics associated with the magnetization reversal process in the sample to be measured. The power of MSHG lies in the ability to separate the vectorial in-plane components through a single measurement. We showed that MSHG is an effective tool to observe the induced precession and the temporal variations in the absolute value of $\mathbf{M}$ for thin film Permalloy induced by short magnetic pulse fields. In addition, this technique may also be used to study the hysteretic properties of magnetic thin films in quasistatic geometries in order to understand the details associated with the low-speed magnetization reversal process.

\section{ACKNOWLEDGMENTS}

The authors would like to thank Tony Kos for assistance in preparing the devices for the pulse-generator measurements. This work was partially supported by the Stichting Fundamenteel Onderzoek der Materie (FOM) and by the European "DYNAMICS" project. This work was also supported by a fellowship within the Postdoctoral Program of the German Academic Exchange Service (DAAD). Work partially supported by NIST, an agency of the U.S. government.

${ }^{1}$ T. J. Silva, P. Kabos, and M. R. Pufall, Appl. Phys. Lett. 81, 2205 (2002).

${ }^{2}$ Th. Gerrits, H. A. M. van den Berg, J. Hohlfeld, L. Bär, and Th. Rasing, Nature (London) 418, 509 (2002).

${ }^{3}$ M. d'Aquinom, W. Scholz, T. Schrefl, C. Serpico, and J. Fidler, J. Appl. Phys. 95, 7055 (2004).

${ }^{4}$ A. Barman, V. V. Kruglyak, R. J. Hicken, J. Scott, A. Kundrotaite, and M. Rahman, J. Appl. Phys. 95, 6998 (2004).

${ }^{5}$ B. Beckmann, U. Nowak, and K. D. Usadel, Phys. Rev. Lett. 91, 187201 (2003); A. Kirilyuk and Th. Rasing, J. Opt. Soc. Am. B 22, 148 (2005).

${ }^{6}$ P. Kabos, A. B. Kos, and T. J. Silva, J. Appl. Phys. 87, 5980 (2000).

${ }^{7}$ T. M. Crawford, C. T. Rogers, T. J. Silva, and Y. K. Kim, IEEE Trans. Magn. 32, 4087 (1996).

${ }^{8}$ Y. Acreman, C. H. Back, M. Buess, O. Portmann, A. Vaterlaus, D. Pescia, and H. Melchior, Science 290, 492 (2000).

${ }^{9}$ B. C. Choi, M. Belov, W. K. Hiebert, G. E. Ballentine, and M. R. Freeman, Phys. Rev. Lett. 86, 728 (2001).

${ }^{10}$ H. F. Ding, S. Pütter, H. P. Oepen, and J. Kirschner, Phys. Rev. B 63, 134425 (2001).

${ }^{11}$ J. W. Lee, J. Kim, S. K. Kim, J. R. Jeong, and S. C. Shin, Phys. Rev. B 65, 144437 (2002).

${ }^{12}$ R. Lopusnik, Ph.D. thesis, University of Kaiserslautern, 2001.

${ }^{13}$ K. H. Bennemann, Nonlinear Optics in Metals (Clarendon, Oxford, 1998), p. 67.

${ }^{14}$ R.-P. Pan, H. D. Wei, and Y. R. Shen, Phys. Rev. B 39, 1229 (1989).

${ }^{15} \mathrm{We}$ can also assume a structure with several magnetic layers. However, in this case the signal does not come from one particular interface.

${ }^{16}$ Th. Rasing, J. Magn. Soc. Jpn. 20, 13 (1996). 